\title{
Correlation of qSOFA with Severity of COVID-19 Infection Assessed on Admission at Tertiary Care Hospital in Lahore
}

\author{
${ }^{1}$ Munaza Javed, ${ }^{1}$ Muhammad Atif Qureshi, ${ }^{2}$ Muhammad Naeem Akhtar, ${ }^{3}$ Saadat Ullah, \\ ${ }^{4}$ Osama Habib, ${ }^{5}$ Muhammad Saqib \\ ${ }^{I}$ Department of Medicine, Azra Naheed Medical College, Superior University, Lahore \\ ${ }^{2}$ Department of, Pulmonology, Lahore General Hospital, Lahore \\ ${ }^{3}$ Department of Medicine, Jinnah Hospital, Lahore \\ ${ }^{4}$ Department of Psychiatry, Azra Naheed Medical College, Superior University, Lahore \\ ${ }^{5}$ Department of Pulmonology, Shaikh Zayed Medical Complex, Lahore
}

\begin{abstract}
Introduction: The COVID-19 crisis is continuously taxing the world with a huge impact on mortality, morbidity and disease related mental stress. COVID-19 has a variable presentation encompassing from simple myalgias to development of ARDS. Early detection of patients with severe COVID 19 infection by utilizing simple bed side clinical tool like qSOFA, which has been previously used for detection of severity of sepsis could help us in early triage and subsequent management of these patients in specialized dedicated units of the hospital. Thus help in limiting the mortality and morbidity because of this deadly contagion. Aims \& Objectives: To correlate the severity of qSOFA score with the clinical severity and outcome of COVID-19 infection at presentation among admitted patients in tertiary care hospitals of Lahore. Place and duration of study: This study was conducted simultaneously at three tertiary care hospitals of Lahore, including Lahore General Hospital, Jinnah Hospital Lahore and Chaudhary Muhammad Akram Teaching and Research Hospital, Lahore. Material \& Methods: A retrospective descriptive study on 120 RT PCR positive confirmed COVID-19 patients of both genders, more than 18 years of age. Case records of patients admitted in General Corona ward, Isolation wards, HDUs and ICUs were analyzed. The data analysis was done in SPSS version 20. Factors including blood pressure, respiratory rate, and conscious level were used to calculate the qSOFA score. COVID 19 infection was divided into mild, moderate, severe and critically ill. The relationship of qSOFA score was studied with severity of COVID-19 infection, outcome of disease and age by using the Pearson correlation. Results: Eighty-four (70\%) patients were male and thirty-six (30\%) were female, with mean age of 59.42 \pm 13.49 years. Sixty-eight, twenty-five, five and 0.8 percent patients had critical, severe, moderate and mild disease respectively. Most frequent comorbids found were hypertension, diabetes, ischemic heart disease. A statistically significant correlation was observed between qSOFA with severity of COVID 19, age, and outcome of infection at the level of 0.01. Conclusion: qSOFA is a reliable bedside tool to measure severity and predict outcome of COVID 19 infection.
\end{abstract}

Key words: COVID 19 infection, qSOFA, disease severity, outcome

\section{INTRODUCTION}

$\mathrm{D}$ ecember 2019 will always be remembered in history as beginning of a pandemic of $21^{\text {st }}$ century. Series of mystical pneumonia cases were reported in individuals linked in some way to an animal market in the city Wuhan, China. This infection spread to the whole world in a short period of time. ${ }^{1}$ A Novel Corona Virus was identified as the cause of this pneumonia and was later named as SARS-CoV-2 virus. $^{2}$ WHO declared it as a pandemic on $11^{\text {th }}$ of March. ${ }^{3}$ The SARS-CoV-2 virus affects all age groups; genders and people from all walks of life. Mode of transmission of this highly contagious virus is through respiratory droplets, contaminated surfaces and contact routes. The incubation period of this virus is 1 to 14 days. ${ }^{4}$ Most of the people start experiencing symptoms on the $6^{\text {th }}$ day after exposure to the virus. ${ }^{5}$

SARS-COV-2 or COVID-19 is an ongoing global catastrophe which may persist for some years to 
come. It has a huge impact on mortality, morbidity and disease related mental stress. Till the month of October, 2020 it has infected over 30 million people worldwide and has a fatality rate of $2.3 \%{ }^{6,7}$ Pakistan detected it first COVID 19 case in start of the year 2020, from which the number of cases have escalated exponentially with the total number crossing 4 lac in just a few months. COVID-19 presents with a myriad of symptoms which are mild in majority of cases, whereas more serious symptoms of pneumonia are experienced in $15 \%$ of the patients. Approximately $5 \%$ of patients develop life threatening complications like pneumonia, sepsis/ disseminated intravascular coagulation (DIC), acute respiratory distress syndrome (ARDS), thromboembolic phenomenon, cardiac injury and acute kidney injury. ${ }^{8}$ These complications are thought to result from damage to the alveolar epithelial cells, activation and dysregulation of innate immune responses thereby ultimately leading to release of pro inflammatory cytokines and chemokines. Thus the development of cytokine storm has an important role in the progression to respiratory and multi-organ failure in COVID-19. ${ }^{9,10}$ Severe COVID-19 disease management therefore requires ICU care, ventilatory and vasopressor support. ${ }^{11,12}$

During this Pandemic a substantial increase in number of COVID-19 patients with sepsis has challenged health care systems worldwide with an overwhelming increase in demand of ICU care and mechanical ventilation. Therefore, a dire need was felt to identify a scoring system which would help in early triage of all patients with COVID-19, so that prompt management could be initiated in dedicated hospital quarters. Quick sequential organ failure assessment score (qSOFA) has come into vogue after recommendations of Sepsis-3 task force in the year 2016 for assessment of prognosis in patients with sepsis. It utilizes three easy to use bedside clinical variables like Glasgow coma scale (GCS), respiratory rate and systolic blood pressure for its calculation. ${ }^{13}$ Consequently qSOFA could be routinely employed as a bedside clinical instrument to categorize the severity of COVID-19 infection and to further guide in prompt patient management. Internationally qSOFA and multiple other scores are being utilized as screening tools for identifying sepsis in patients, and in further predicting their outcomes. Therefore, in this retrospective study we wanted to assess the reliability of qSOFA score in our local Pakistani setup in predicting severity of COVID 19 infection through coorelation with clinical severity as well as its outcome. By using this simple bedside scoring system, critically ill patients could be triaged early and ultimately managed effectively.

\section{MATERIAL AND METHODS}

This retrospective descriptive study was conducted at three tertiary care hospitals of Lahore including Lahore General Hospital, Jinnah Hospital Lahore and Chaudhary Muhammad Akram Teaching and Research Hospital, Lahore. After taking approval from the Institutional Ethical Review Board, hospital records of 120 patients of either gender were thoroughly reviewed in the study. COVID-19 confirmed case was defined as a patient with positive RT-PCR for SARS-CoV-2 in either nasal or pharyngeal swab specimens. All COVID-19 RTPCR positive patients of more than 18 years of age were included in the study whereas all COVID-19 RT- PCR negative patients or who were less than 18 years of age were excluded. The requirement for informed consent was waived because of the retrospective study design. Confidentiality of record was maintained and details were only disclosed to the authors.

Case records 120 inpatients of General Corona ward, Isolation wards, HDUs and ICUs were analyzed and recorded on a preformed questionnaire in first phase. Quick Sequential Organ Failure Assessment (qSOFA) score on admission was calculated (Glasgow coma scale $<15$, systolic blood pressure $<100 \mathrm{mmHg}$ and respiratory rate $>22$ breath/min) as $0,1,2$ and $3 .{ }^{13}$ COVID 19 infection was classified as mild, moderate, severe and critical according to Pakistan Ministry of Health clinical management guidelines of COVID 19. ${ }^{14}$ Outcome of the disease was categorized into two groups first as discharge from hospital after recovery with no need of supplementary oxygen and secondly as death of the patient during hospital stay.

\section{Statistical analysis:}

In the second phase the data was entered into Statistical Package for Social Sciences (SPSS) version 20 for statistical analysis. The data contained both qualitative and quantitative variables. Quantitative data like age was expressed as mean $\pm \mathrm{SD}$ whereas qualitative data like gender, Severity of COVID-19 infection and qSOFA score were presented in the form of frequency and percentage. Relationship between qSOFA at presentation with severity of COVID 19 infection and outcome were studied by applying the Pearson coefficient. 


\section{RESULTS}

Seventy percent of our patients were male while thirty percent were female. Mean age calculated was 59.42 years \pm 13.49 (range from 27 to 94 years). Seventy seven percent of patients were more than 51 years of age. One patient $(0.8 \%)$ patients in our study had a mild disease whereas $68.3 \%, 25.8 \%$ and $5 \%$ had critical, severe and moderate disease respectively (Table-1). Commonest comorbids reported were HTN followed by diabetes and ischemic heart disease. One hundred and five $(87.5 \%)$ of our patients were discharged from the hospital whereas fifteen $(12.5 \%)$ died during the hospital stay.

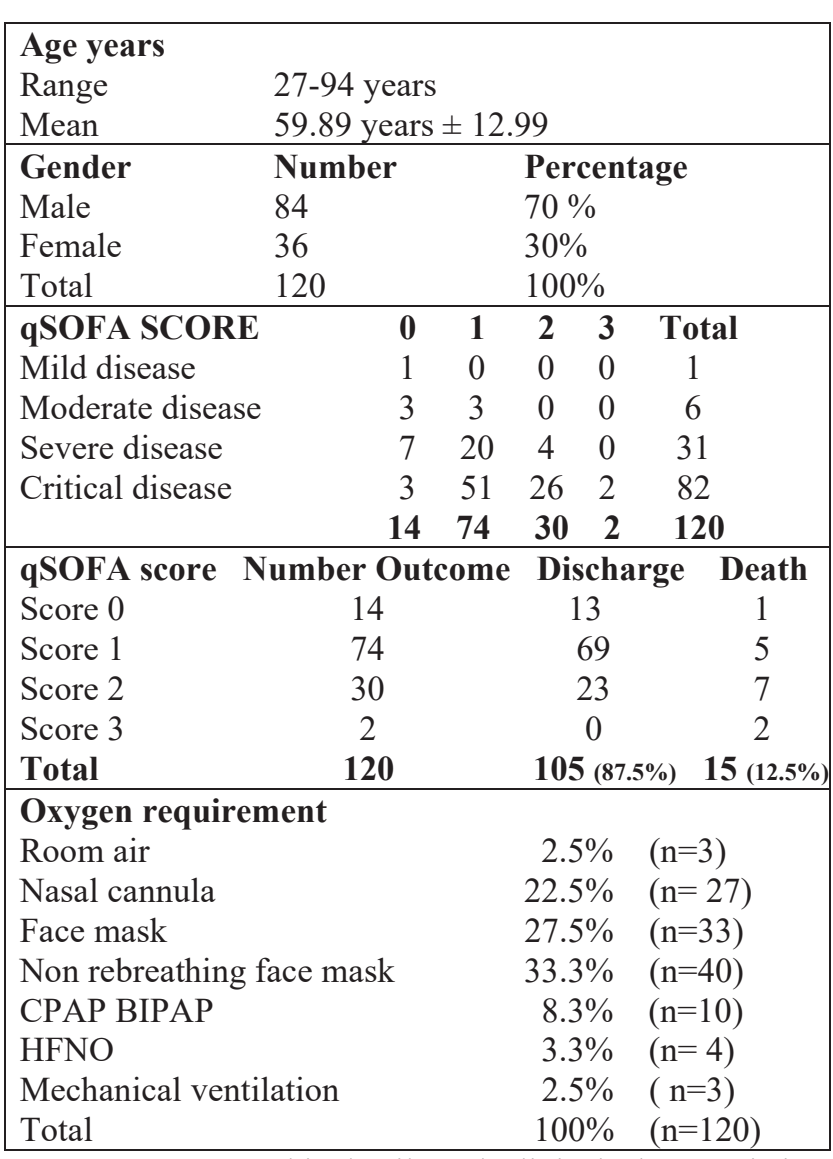

Table-1: Demographic details and Clinical Characteristics of COVID 19 infection ( $\mathrm{n}=120$ patients)

One hundred and seventeen $(97.5 \%)$ of our patients required supplemental Oxygen or artificial ventilation, whereas only three $(2.5 \%)$ of our patients maintained their oxygen on room air. The qSOFA score of $0,1,2$ and 3 was found in 14, 74, 30 , and 2 of the patients respectively. All patients $100 \%(n=2)$ with qSOFA score of 3 in our study had a critical disease and died during hospital stay. On applying the two tailed Pearson coefficient it was statistically significant at the level of 0.01 between
qSOFA and severity of COVID-19 infection (Table-2).

\begin{tabular}{|c|l|c|c|}
\hline \multicolumn{2}{|c|}{} & Severity & qSOFA \\
\hline \multirow{4}{*}{ Severity } & Pearson Correlation & 1 & $.435^{* *}$ \\
\cline { 2 - 4 } & Sig. (2-tailed) & & .000 \\
\cline { 2 - 4 } & $\mathrm{N}$ & 120 & 120 \\
\hline \multirow{4}{*}{ qSOFA } & Pearson Correlation & $.435^{* *}$ & 1 \\
\cline { 2 - 4 } & Sig. (2-tailed) & .000 & \\
\cline { 2 - 4 } & $\mathrm{N}$ & 120 & 120 \\
\hline ** Correlation is significant at the 0.01 level (2-tailed). \\
\hline
\end{tabular}

Table-2: Pearson Correlation of qSOFA with Severity of infection

\begin{tabular}{|l|l|c|c|}
\hline \multicolumn{2}{|c|}{} & qSOFA & outcome \\
\hline \multirow{4}{*}{ qSOFA } & Pearson Correlation & 1 & $.297^{* *}$ \\
\cline { 2 - 4 } & Sig. (2-tailed) & & .001 \\
\cline { 2 - 4 } & $\mathrm{N}$ & 120 & 120 \\
\hline \multirow{4}{*}{ outcome } & Pearson Correlation & $.297^{* *}$ & 1 \\
\cline { 2 - 4 } & Sig. (2-tailed) & .001 & \\
\cline { 2 - 4 } & $\mathrm{N}$ & 120 & 120 \\
\hline
\end{tabular}

**. Correlation is significant at the 0.01 level (2-tailed).

Table-3: Pearson Correlation of qSOFA with disease outcome

Similarly Pearson coefficient was statistically significant at the level of 0.01 for qSOFA score with disease outcome (Table-3) as $60 \%(\mathrm{n}=9)$ of our patients who died during the hospital stay had a qSOFA score of 2 or above. Similarly Pearson coefficient was statistically significant for age and mode of Oxygenation. Both hypertension and diabetes when occurring in the same patient had a significant correlation with qSOFA score combined but was insignificant when studied separately (Table-4).

\begin{tabular}{|c|c|}
\hline Variables & $\begin{array}{c}\text { Pearson's Correlation } \\
\text { Level of Significance }\end{array}$ \\
\hline $\begin{array}{c}\text { qSOFA and Disease } \\
\text { Severity }\end{array}$ & $\begin{array}{c}\text { Correlation is significant at } \\
\text { the } 0.01 \text { level }\end{array}$ \\
\hline $\begin{array}{c}\text { qSOFA and Mode of } \\
\text { Oxygenation }\end{array}$ & $\begin{array}{c}\text { Correlation is significant at } \\
\text { the } 0.01 \text { level }\end{array}$ \\
\hline $\begin{array}{c}\text { qSOFA and Disease } \\
\text { Outcome }\end{array}$ & $\begin{array}{c}\text { Correlation is significant at } \\
\text { the } 0.01 \text { level }\end{array}$ \\
\hline qSOFA and DM+HTN & $\begin{array}{c}\text { Correlation is significant at } \\
\text { the } 0.01 \text { level }\end{array}$ \\
\hline qSOFA and Age & $\begin{array}{c}\text { Correlation is significant at } \\
\text { the } 0.05 \text { level }\end{array}$ \\
\hline
\end{tabular}

Table-4: Pearson Correlations 


\section{DISCUSSION}

World has faced the epidemics of SARS and MERS in the past but there is no comparison to the magnitude in which COVID-19 has affected the world as it has led to segregation and isolation of billions of people. ${ }^{15}$ Severe COVID-19 leading to ARDS requires ICU care and mechanical ventilation. In hospitalized COVID-19 patients, the prevalence of hypoxemic respiratory failure is around $20 \%$, and more than $25 \%$ of them may require intensive care treatment. ${ }^{16}$

The age range in our study was 27-94 years; of which most of the patients were around 60 years of age Our findings were similar to the findings of a Chinese study who reported $76.1 \%$ of their patient as being more than 65 years of age. ${ }^{17,18}$ Similar to the findings of Wang D we found a significant correlation between the qSOFA score on admission with age of patients. The male predominance in our study was supported by the findings of $\mathrm{Ru}$ et al who reported $68.8 \%$ of their patients as being male. ${ }^{19,20}$ Increased exposure of males to the outside environment for work and other needs of daily life could be one of the reasons for this high infection rate in them. Findings of an Egyptian study were contrary to our findings as they reported a slight female predominance in their study. ${ }^{21} \mathrm{We}$ found no significant correlation between severity of infection and gender on applying the Pearson's Correlation which was contradictory to the findings of $\mathrm{Ru}$ et al who had reported a severe disease in male patients.

Our study found a direct correlation between the severity of COVID-19 infection and qSOFA score at presentation, as the qSOFA score increased so did the severity of COVID 19 infection. None of our patients with a qSOFA of 2 or 3 had any mild or moderate disease, almost all of them had either severe or critical disease. Our findings are in concordance with the findings of Almazeedi et al who in their study of 1096 COVID 19 patients, reported a high qSOFA score to be one of the factors which lead to a severe disease followed by admissions in the intensive care units. ${ }^{22}$ Similarly we found a direct relationship between high qSOFA score and grave outcome (death) in our study as $100 \%(n=2)$ of our patients who had a qSOFA score of 3 died during hospital stay. Most of the patients who died in our study had a qSOFA $>1$. Our findings were supported by an Iraqi study who had reported qSOFA $>1$ to be a risk factor for the need of ICU care, mechanical ventilation and death. Therefore, a qSOFA score of $>1$ was inversely associated with survival and had a grave prognosis. Similarly Freund et al proposed a mortality rate of $3 \%$ for patients with a qSOFA $>1$ as compared to the $24 \%$ for patients with qSOFA $>2 .{ }^{23}$

On the contrary Marion Ferreira et al have declared qSOFA score to be of little use in identifying patients with COVID-19 infection associated sepsis as the underlying mechanisms are very different from sepsis due to an underlying bacterial infections and include endothelial injury and development of micro thrombi as the main pathogenic mechanism in COVID-19. ${ }^{24}$ Similar sub optimal performance of qSOFA score has been reported by Ackermann M et al who found that a qSOFA score $>1$ was found in patients requiring ICU care due to ARDS, however no significant difference was found in qSOFA score in critically ill COVID-19 patients requiring mechanical ventilation from those who did not require ventilatory support. ${ }^{25,26}$

\section{CONCLUSION}

A direct correlation was found between qSOFA score on admission with severity of COVID-19 infection, age and outcome of disease.

\section{Limitations:}

We did not study the qSOFA score relation with need of ICU care and a comparison between effectiveness of different disease severity prognostic models like CALL, NEWS, CRB65 and CURB65 was not made. Further research in this regard could elaborate our understanding and help us in effectively managing this rapidly evolving health crisis.

\section{REFERENCES}

1. Tu H, Tu S, Gao S, Shao A, Sheng J. The epidemiological and clinical features of COVID-19 and lessons from this global infectious public health event. $\mathrm{J}$ Infect doi:10.1016/j.jinf.2020.04.011 .

2. Zhu N, Zhang D, Wang W, Li X, Yang B, Song $\mathrm{J}$, et al. A novel coronavirus from patients with pneumonia in China, 2019. New England Journal of Medicine. 2020; 382:727-733.

3. World Health Organization. WHO Characterizes COVID-19 as a Pandemic 11March 2020.

4. Guo YR, Cao QD, Hong ZS, Tan YY, Chen $\mathrm{SD}$, Jin $\mathrm{HJ}$ et al . The Origin, Transmission and Clinical Therapies on Coronavirus Disease 2019 (COVID-19) Outbreak - An Update on the Status- Mil Med Res. 2020 Mar 13;7(1):11.

5. Tiana S, Hub N, Loua J, Chenc K, Kanga X, Xiang Z Characteristics of COVID-19 infection 
in Beijing / Journal of Infection 80 (2020) 401$4061 \mathrm{~S}$.

6. $\mathrm{Wu} \mathrm{Z}$, McGoogan JM. Characteristics of and important lessons from the coronavirus disease 2019 (COVID-19) outbreak in China: summary of a report of 72314 cases from the Chinese Center for Disease Control and Prevention. Jama. 2020 Apr 7; 323(13):1239-42.

7. European Centre for Disease Prevention and Control COVID-19: Situation update worldwide Accessed September 10, 2020 at https://www.ecdc.europa.eu/en/geographicaldistribution-2019-ncov-cases.

8. Novel Coronavirus Pneumonia Emergency Response Epidemiology Team. The Epidemiological Characteristics of an Outbreak of 2019 Novel Coronavirus Diseases (COVID19) in China 2020; 41(2):145-151.

9. Zhang C, Wu Z, Li JW, Zhao H, Wang GQ. Cytokine release syndrome in severe COVID19: interleukin-6 receptor antagonist tocilizumab may be the key to reduce mortality. Int J Antimicrob Agents 2020; 55: 105954.

10. Channappanavar R, Perlman S. Pathogenic human coronavirus infections: causes and consequences of cytokine storm and immunopathology. Semin Immunopathol 2017; 39: 529-39

11. Cevik M, Bamford GG, Ho A COVID-19 pandemic - a focused review for clinicians Clin Microbiol Infect. 2020 Jul; 26(7): 842-7.

12. Wang D, Hu B, Hu C, Zhu F, Liu X, Zhang J et al. Clinical characteristics of 138 Hospitalized patients with 2019 novel coronavirus-infected pneumonia in Wuhan, China. JAMA. 2020; 323:1061-1069.

13. Singer M, Deutschman CS, Seymour CW, et al. The third international consensus definitions for sepsis and septic shock (Sepsis-3). JAMA. 2016; 315:801-10.

14. Government of Pakistan Ministry of National Health Services Regulations \& Coordination Clinical Management Guidelines for COVID-19 Infections available from:

http://covid.gov.pk/new_guidelines/05June2020 20200106_Clinical_Management_Guidelines for_COVID-19_infection_v2.pdf clinical spectrum

15. Sandford A. Coronavirus: Half of humanity now on lockdown as 90 countries call for confinement. Accessed on. 2020 Apr; 17.

16. European Centre for Disease Prevention and Control COVID-19: Situation update worldwide Accessed September 10, 2020 at https://www.ecdc.europa.eu/en/geographicaldistribution-2019- ncov-cases.

17. Grasselli G, Zangrillo A, Zanella A, Antonelli M, Cabrini L, Castelli A, et al. Baseline characteristics and outcomes of 1591 patients infected with SARS-CoV-2 admitted to ICUs of the lombardy region, Italy. JAMA 2020 Apr 6.

18. Wang D, Yin Y, Hu C, Liu X, Zhang X, Zhou S, et al. Clinical course and outcome of 107 patients infected with the novel coronavirus, SARS-CoV-2, discharged from two hospitals in Wuhan, China. Crit Care. 2020.Apr30;24(1):188.

19. Bhatraju PK, Ghassemieh BJ, Nichols M, et al. Covid-19 in critically ill patients in the Seattle region - case series. N Engl J Med. 2020.

20. Du RH, Liu LM, Yin W, Wang W, Guan LL, Yuan ML et al. Hospitalization and critical care of 109 decedents with COVID-19 pneumonia in Wuhan, China. Ann Am Thorac Soc. 2020.

21. Hussein AM, Galal I, Mohamed MMAR, Ibrahim MEAA, Ahmed SB Survival and 30days hospital outcome in hospitalized COVID19 patients in Upper Egypt: Multi-center study. https://doi.org/10.1101/2020.08.26.20180992

22. Almazeedia S, Youhaa S, Jamal MH, Haddada MA, Muhainia AA, Ghimlas FA et al Characteristics, risk factors and outcomes among the first consecutive 1096 patients diagnosed with COVID-19 in Kuwait E Clinical Medicine, Volume 24, July 2020, Pages 100462

23. Freund Y, Lemachatti N, Krastinova E, Marie VL, Erick CY, Aurélie A, et al. Prognostic accuracy of sepsis-3 criteria for in-hospital mortality among patients with suspected infection presenting to the emergency department. JAMA. 2017; 317:301-8.

24. Ferreira M, Blin T, Collercandy N, Szychowiak P, Dequin PF, Jouan Yet al Critically ill SARS-CoV-2-infected patients are not stratified as sepsis by the qSOFAFerreira et al. Ann. Intensive Care (2020) 10:43

25. Ackermann M, Verleden SE, Kuehnel M, Haverich A, Welte $\mathrm{T}$, Laenger $\mathrm{F}$, et al. Pulmonary Vascular Endothelialitis, Thrombosis, and Angiogenesis in Covid-19. N Engl J Med 2020.

26. Wang Y, Lu X, Chen H, Chen T, Su N, Huang F, et al. Clinical course and outcomes of 344 intensive care patients with COVID-19. Am J Respir Crit Care Med. 2020. 
The Authors:

Dr. Munaza Javed

Associate Professor,

Department of Medicine,

Azra Naheed Medical College,

Superior University, Lahore.

Dr. Muhammad Atif Qureshi

Professor of Medicine,

Department of Medicine,

Azra Naheed Medical College,

Superior University, Lahore.

Dr. Muhammad Naeem Akhtar

Senior Registrar,

Department of Pulmonolgy,

Lahore General Hospital, Lahore.

Dr. Saadat Ullah

Senior Registrar Medical Unit 1, Jinnah Hospital, Lahore saadatullah44@gmail.com
Dr. Osama Habib

Associate Professor

Department of Psychiatry,

Azra Naheed Medical College

Superior University, Lahore

Dr. Muhammad Saqib

Assistant Professor,

Department of Pulmonology,

Shaikh Zayed Medical Complex, Lahore

03004479679

dr.saqib.ch@gmail.com

\section{Corresponding Author:}

Dr. Munaza Javed

Associate Professor,

Department of Medicine,

Azra Naheed Medical College,

Superior University, Lahore.

E-mail: munaza_mji@yahoo.com 